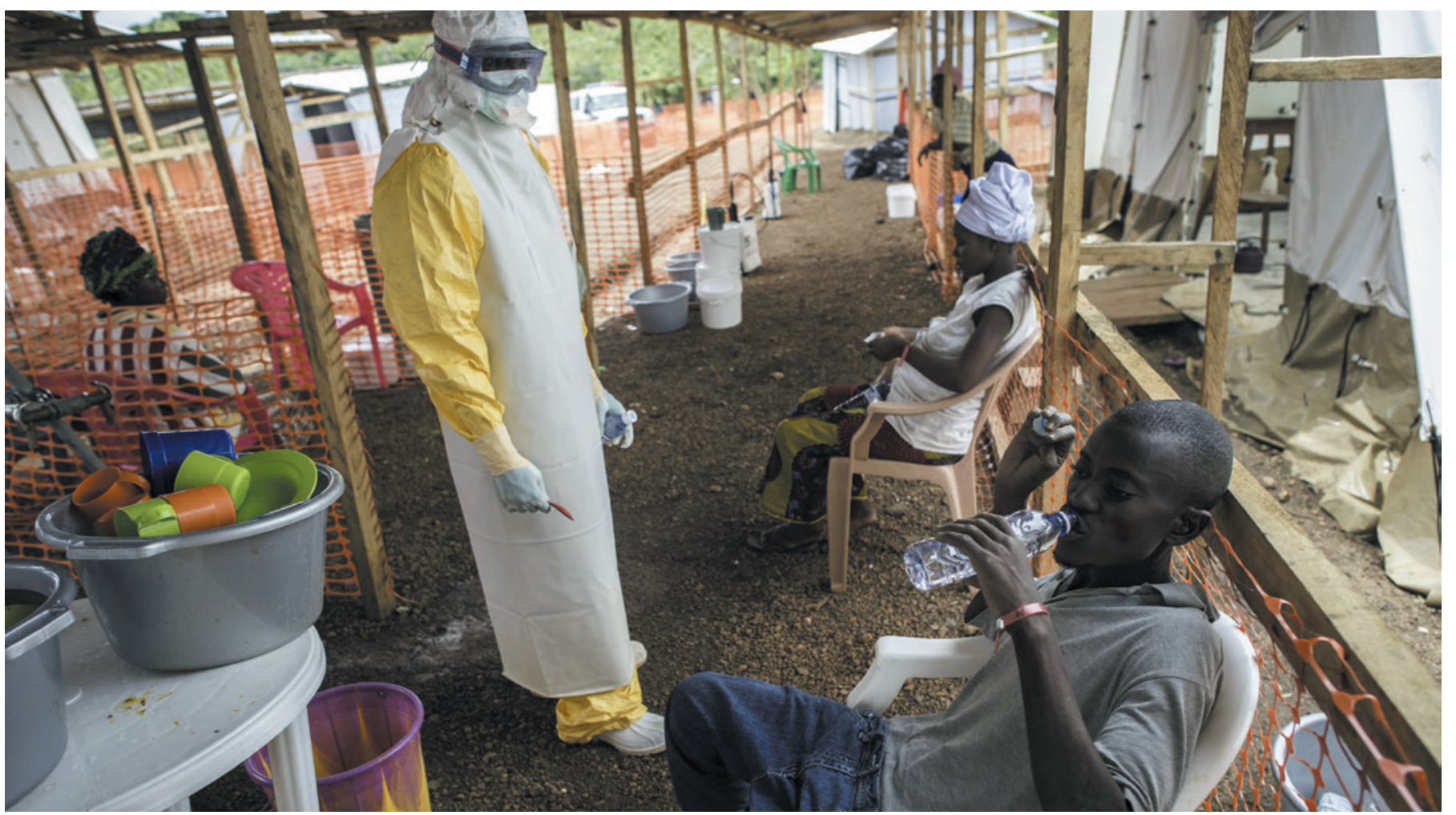

A nurse attends to a patient at a Médecins Sans Frontières Ebola treatment centre in Kailahun, Sierra Leone.

\title{
Tough choices to reduce Ebola transmission
}

\section{Christopher J. M. Whitty and colleagues explain why the United Kingdom is funding many small community centres to isolate suspected cases in Sierra Leone.}

$\mathrm{T}$ he Ebola outbreak in West Africa is larger than all previous Ebola outbreaks combined, and is still expanding ${ }^{1}$. With a death toll in the thousands, and mortality at around $70 \%$, it has undermined fragile health-care systems by filling hospitals with highly infectious patients and killing health workers.

Transmission in dense urban populations presents challenges never before seen. Vaccines and drugs would offer a complementary approach to control and they must be fast tracked. But these medicines are not available for immediate widespread use. To quell this unprecedented outbreak, we have to use methods that have not been tested.

Experience from past outbreaks has established reliable methods to control transmission in hospitals and at funerals of people who die from Ebola - two of the three main venues for transmission (see 'Hospitals and funerals'). These efforts remain essential, and the scientific and operational strands must complement one another.
What most distinguishes the current situation from previous outbreaks is the high proportion of transmission occurring in the community. No techniques have been developed to control community transmission at this scale $e^{2,3}$. Notwithstanding uncertainties, the global community must act now.

The UK government is leading the international response to Ebola in Sierra Leone, providing technical, financial and logistical help. This article sets out the scientific basis for the UK government's strategy to assist Sierra Leone's government to reduce transmission. In addition to substantially scaling up conventional capacities at hospitals, we plan to help to build and support community isolation centres where people can voluntarily come to be isolated if they suspect that they have the disease.

These interventions represent uncertain ground, but hesitation is more dangerous than trying out potentially ineffective methods (see 'Delays mean more deaths'). Climbing rates of infection could soon overwhelm
Sierra Leone's already challenged health services, and so remove any realistic chance of public-health intervention ending the outbreak. The Sierra Leonean health system and the many brave local and international health staff need help to lower transmission to the point at which mopping up remaining islands of high transmission will be possible.

Until the outbreak is over, we will not know whether we have launched the best response. We invite critiques and suggestions, but must act swiftly. Further delay will result in more infections and deaths, and only sabotage future efforts.

\section{THE CHALLENGE}

About 500 new cases of Ebola are being diagnosed every week. This number is expected to double in the next 30 days $^{4}$. The force of transmission of a disease outbreak is quantified as $R$, the average number of people infected by each newly infected person (the reproduction number). When $R=1$, an outbreak is neither increasing nor decreasing. 
EBOLA TRANSMISSION

\section{Hospitals and funerals}

The initial symptoms of Ebola (which may include fever, diarrhoea and vomiting) mimic those of many common diseases, including malaria, pneumonia and gastrointestinal infections. Transmission occurs through bodily fluids: diarrhoea, vomit, blood and probably sweat and semen. Patients become infectious when they are symptomatic, and remain highly infectious until they begin to recover. Corpses are highly infectious.

Controlling transmission requires minimizing contact with bodies, bodily fluids and contaminated items such as mattresses, clothes and clinical waste. Transmission does not occur through breathing or insects. Transmissibility through existing modes of infection could evolve as the outbreak expands.
Ending previous Ebola outbreaks relied on stopping transmission in hospitals through strict infection control; adapting funeral rites to reduce risk of transmission; helping local people to protect themselves; and actively tracking down those likely to be infected ${ }^{7}$.

Infection control in hospitals requires sufficient resources and trained personnel to implement measures, such as using personal protective equipment. In relatively well-equipped hospitals with stringent training and control protocols (such as those run by Médecins Sans Frontières), infection rates remain low.

Reducing transmission from the dead is more complex, and social scientists play an essential part. Protocols for handling and disposing of a body to minimize the risk of infection are well established, but must be implemented with the cooperation and consent of grieving communities. Funeral rites often require that family members and mourners have extensive contact with the deceased.

Previous Ebola outbreaks in the Democratic Republic of Congo and Uganda have shown that unless infection-control routines can be adapted to local burial practices, communities will continue their traditions. Anthropologists have documented funeral rites in Sierra Leone and other countries. Dialogues with Ebolastricken communities are essential to adapting their traditions to reduce body handling respectfully.
According to our analyses, $R$ in Sierra Leone is currently between 1.2 and 1.5 . In some areas, $R$ is considerably higher. If $R$ remains above 1 , any public-health intervention (except a vaccine) will eventually be overwhelmed by the number of new infections. Getting $R$ below 1 is the single strategic aim of the UK effort at this stage of the outbreak.

Reducing $R$ depends on stalling transmission in the community, which depends on reducing the time between when people first show symptoms and are isolated. Delay occurs in two broad settings: patients who have symptoms consistent with Ebola but have not been positively diagnosed; and people diagnosed with Ebola who are being nursed at home because of reticence to move to facilities, or a lack of available health-care beds or transport. Patients in the later stages of Ebola are the most infectious, in part because symptoms such as vomiting and diarrhoea are worse ${ }^{5}$. Models vary, but most work, including that led by N.F. and W.J.E., suggests that isolating $70 \%$ or more of Ebola cases within three days of their becoming symptomatic is key to reducing $R$ to 1 .

\section{TWO-PRONGED STRATEGY}

Designing measures to control community transmission poses epidemiological, clinical and social-science challenges. Currently, most people with early Ebola-like symptoms will not have Ebola, but diseases such as malaria and pneumonia. Undiagnosed individuals should be isolated both from health workers (as in Ebola wards) and from each other, so that the true Ebola cases do not infect those with other diseases. The high number of suspected cases greatly complicates isolation efforts.

Community control of previous Ebola outbreaks relied on active 'case-finding': tracing contacts of known cases and then monitoring and quickly isolating suspected cases early. It can take as many as ten staff to monitor the contacts of just a single case, and the task is potentially more difficult in mobile urban populations, especially as Ebola case numbers rise.

The ideal approach would be active casefinding combined with isolating patients in fully equipped and staffed hospitals, but this is not practical in the current situation.

"Centres
must be able
to operate
with small,
relatively
unspecialized
staff drawn
from the
community."
In some areas, the outbreak has already overrun hospitals; many suspected Ebola cases are being turned away for lack of beds. An unknown proportion of cases remain at home. For those who seek medical care, the current median time between becoming symptomatic (and thus infectious) and isolation in Sierra Leone is four days. Many wait more than a week.

These delays will only get longer. Current UK aid efforts - which have so far pledged more than $£ 200$ million (US\$320 million) and the largest UK troop deployment outside Afghanistan - will help to increase Sierra Leone's bed capacity threefold by January. But at current measures of $R$, the projected increase in new cases (thousands per week) will far exceed the number of possible new hospital beds. To avoid that scenario, beginning this month, affected regions must substantially increase rates of early isolation for suspect and confirmed Ebola cases.

One proposed strategy — giving families information and basic personal protective equipment (PPE) to minimize transmission while nursing patients at home - is problematic. Using PPE safely is difficult even for professionals, as infection rates in health-care workers demonstrate. And identifying cases and training families requires staff that Sierra Leone does not have. This approach is acceptable only as a desperate humanitarian measure when there is no space available in health facilities. It is not a good strategy to reduce transmission.

\section{PASSIVE CASE-FINDING}

The UK government has decided to support another strategy: passive case-finding with community isolation. This is at the request of the government of Sierra Leone and endorsed by the World Health Organization. The strategy, also called voluntary sequestration, encourages those with suspected Ebola infection to gather in units where they can receive basic health care and avoid infecting members of their families and communities. By quickly isolating suspected cases (and boosting the proportion of such cases isolated early), this approach aims to keep people with Ebola from infecting others, until $R$ is pulled below 1 .

Some models are currently being piloted near Sierra Leone's capital, Freetown, codesigned with local clinicians and communities. An initial 200 community units can be expanded according to how and where the outbreak progresses. All share similar requirements. Community-level units must be easy to construct, relying mainly on tents or repurposed buildings in or near affected communities, that can be set up in days or weeks. Many small units are better than a few large ones. They can be placed closer to communities (distance can be a barrier to people 


\section{DELAYS MEAN MORE DEATHS}

For every week that effective interventions are not implemented in Ebola-stricken areas, the case-number peak will be pushed higher and later, meaning that more hospital beds will be needed.

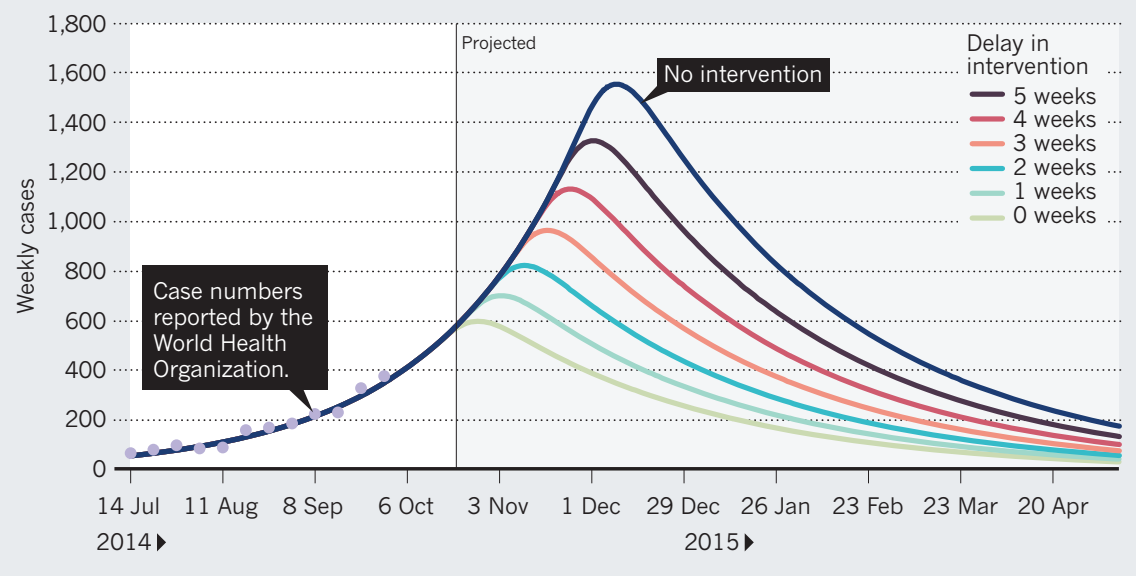

checking themselves in). It also reduces the risk of a major outbreak if infection control breaks down in one unit. Centres must be able to operate with small, relatively unspecialized staff drawn from the community, supported with basic training and provision of equipment and supplies by agencies such as non-governmental organizations (NGOs).

Most importantly, centres must not become sources of new infections. This means effective isolation, as well as diagnosing and moving confirmed Ebola cases on to specialized treatment facilities as quickly as possible.

Because most patients in the early stages of the disease will have relatively minor symptoms, one health worker can care for several people. And because patients choose to report to these centres, communities are more likely to accept them. Even incomplete coverage could yield successes. The aim is not to find every suspected case, but to attract enough early cases to shorten the time that infectious people spend in the community, and so reduce $R$.

\section{DATA, MODELLING AND RISK}

There is little current evidence from previous outbreaks on how well, or even whether, voluntary community isolation reduces transmission. We will need to learn and adjust as data come in. Accurate, recent, open data are essential to shape an effective response, but data-capture systems were implemented only in September in the worst-affected countries. These now allow us to estimate $R$ more accurately and to track time between symptoms and isolation.

Major uncertainties remain - most notably around the proportion of Ebola cases currently being detected by health systems. If only a small proportion of cases are being identified, then a dramatic increase in self-reporting is essential. Conversely, if the major issue is, as we suspect, delays in isolation, then even a one- or two-day reduction in that time might be enough to achieve control.

Modelling can help to predict what provisions are needed. Our current projections of the outbreak in Sierra Leone suggest that more than 200 ten-bed isolation units will be required by the end of December. This estimate may be too low.

The UK government has been incorporating social-science advice into the incentives for bringing sick patients to community centres ${ }^{6}$. The desire for good medical care, for protecting loved ones from infection and possibly provision of food or money would all encourage people with early symptoms to go to Ebola evaluation centres. But distance, suspicion, fear and stigma will discourage early reporting. Under-incentivizing would mean that too few people with Ebola will report to centres to reduce transmission. Over-incentivizing could mean that too many non-infected people report, overwhelming facilities and increasing risks of transmission. To achieve the optimal balance, facility planners will need to engage with local populations and be flexible enough to adopt better practices as evidence accumulates.

Careful collaborative work with communities, building on what is already known from anthropological studies and field reconnaissance, must guide how facilities are located, designed, staffed and operated to maximize social acceptance.

This public buy-in is essential. Communities have reacted negatively to the opening of treatment facilities, and in some cases, people have forcibly removed family members from isolation units. Rumours of nefarious killing and body-part theft have circulated, reflecting anxieties that resonate with people's experiences of disease, war and government intervention (see www.ebola-anthropology.net). The murder of health workers and journalists in Guinea this September exemplifies the risks to staff if interventions go wrong.
Optimal design will be learned on the fly. We know how to minimize infection in dedicated Ebola wards with highly trained and supervised staff, but not in facilities with lightly trained staff in which most people do not have Ebola (but some do). Evidence will have to be collected on the best ways to design units, place patients, speed Ebola diagnoses and transport cases. There are likely to be early failures from which the global community must learn. Given the risks that health workers take, getting Ebola vaccines to staff working in the units as soon as they have proved safe is an ethical imperative.

Extrapolating from data collected in neighbouring countries on common pathogens and antimicrobial drug resistance will be essential. Diagnostic testing, except for Ebola, must be kept to a minimum to avoid contamination from blood and sharp instruments. Health workers should use treatments on the basis of symptoms alone to deliver appropriate care for malaria and bacterial disease without testing, a practice that has been used effectively in many under-resourced settings. Faster and safer Ebola tests (those that would not require using blood with the live virus) would substantially improve clinical management.

We hope that in the coming months, safe and effective vaccines will be produced at large enough volumes to stop this outbreak, but thousands are dying now. Qualitative, quantitative and clinical sciences, and the ability to adapt and learn from mistakes, are urgently needed to reduce community transmission.

Christopher J. M. Whitty is chief scientific adviser at the UK Department for International Development. Jeremy Farrar is director of the Wellcome Trust in London, UK. Neil Ferguson is professor of mathematical biology at Imperial College London, UK. W. John Edmunds is professor of infectious-disease modelling at the London School of Hygiene \& Tropical Medicine, UK. Peter Piot is director of the London School of Hygiene \& Tropical Medicine, UK. Melissa Leach is director of the Institute for Development Studies in Brighton, UK. Sally C. Davies is chief medical officer and chief scientific adviser at the UK Department of Health. e-mail:c-whitty@dfid.gov.uk

1. WHO Ebola Response Team N. Engl. J. Med. 371, 1481-1495 (2014).

2. World Health Organization Ebola Response Roadmap Situation Report: 31 October 2014 (WHO, 2014)

3. Kerstiëns, B. \& Matthys, F. J. Infect. Dis. 179 (Suppl. 1), S263-S267 (1999).

4. Borchert, M. et al. BMC Infect. Dis. 11, 357 (2011)

5. Bausch, D. G. et al. J. Infect. Dis. 196 (Suppl. 2), S142-S147 (2007).

6. Hewlett, B. S., Epelboin, A., Hewlett, B. L. \& Formenty, P. Bull. Soc. Pathol. Exot. 98, 230-236 (2005).

7. Heymann, D. L. Nature 514, 299-300 (2014). 